\title{
Emergence agitation/delirium: we still don't know
}

\author{
Kyung Hwa Kwak \\ Department of Anesthesiology and Pain Medicine, School of Medicine, Kyungpook National University, Daegu, Korea
}

First described in the early 1960s, emergence agitation is a clinical condition in which children experience a variety of behavioral disturbances, including crying, sobbing, thrashing, and disorientation, during early emergence from anesthesia [1]. Although emergence agitation is not a new phenomenon in clinical practice, it is of growing interest because its incidence appears to be increasing with the widespread use of sevoflurane.

Agitation is a state of mild restlessness and mental distress. Agitation can arise from any number of sources, including pain, physiological compromise, or anxiety [2]. Sikich and Lerman [3] defined emergence delirium as "a disturbance in a child's awareness of and attention to his/her environment with disorientation and perceptual alterations, including hypersensitivity to stimuli and hyperactive motor behavior in the immediate postanesthesia period." Emergence agitation and emergence delirium have been used interchangeably in most of the literature.

Despite numerous attempts to explain the causes of emergence agitation/delirium, there is no definitive explanation. Possible causes include pain, preoperative anxiety, type of surgical procedures, personal characteristics of the patient, and type of anesthetics, although no sole factor can explain the etiology of emergence agitation/delirium [4], in large part due to the greatest obstacle to understanding emergence agitation/delirium is the lack of a standardized tool to assess it. The reported prevalence of emergence agitation/delirium varies greatly in the literature, ranging from $10 \%$ to $80 \%$ $[5,6]$, depending on the definition and criteria of emergence agitation/delirium. Sikich and Lerman [3] recently developed the Pediatric Anesthesia Emergence Delirium (PAED) scale, which incorporated cognitive-related assessment items in addition to agitation behaviors, and were able to provide more reliability and validity than other scores. However, they did not provide a validated threshold value in the PAED scale to indicate emergence agitation/delirium. Comparing study results is therefore difficult because of the differences in definitions and the variety of unvalidated scales.

Various attempts have been made to reduce the problem of emergence agitation/delirium. Many drugs, including propofol, fentanyl, clonidine and dexmedetomidine, have been investigated to attenuate the incidence of emergence agitation/delirium [7]. In this issue of the Korean Journal of Anesthesiology, Lee et al. [8] studied the clinical usefulness of a single dose of propofol $(1 \mathrm{mg} / \mathrm{kg})$ at the end of adenotonsillectomy for reducing the incidence and severity of emergence agitation/delirium after sevoflurane anesthesia, but found no effect. It has been known that propofol delays or modifies emergence and could decrease the incidence of emergence agitation/delirium. However, the rapid pharmacokinetics of propofol and the relatively low doses given $(1 \mathrm{mg} / \mathrm{kg})$ could explain the failure of bolus doses to prevent emergence agitation/delirium [9]. Other doses of propofol should be tested at emergence to clarify its role in preventing emergence agitation/delirium. One flaw of the Lee et al. [8] study is the difficulty in discriminating between agitation caused by postoperative pain and emergence agitation; a limitation discussed by the authors. They measured the presence of emergence agitation using Aono's four point scale and the severity of emergence agitation using the PAED scale. The definition of emergence agitation in their study included children who showed inconsolable crying as well

Corresponding author: Kyung Hwa Kwak, M.D., Department of Anesthesiology and Pain Medicine, School of Medicine, Kyungpook National University, 50, Samdeok-dong 2-ga, Jung-gu, Daegu 700-721, Korea. Tel: 82-53-420-5864, Fax: 82-53-426-2760, E-mail: kwakkh@knu.ac.kr (c) This is an open-access article distributed under the terms of the Creative Commons Attribution Non-Commercial License (http:// creativecommons.org/licenses/by-nc/3.0/), which permits unrestricted non-commercial use, distribution, and reproduction in any medium, provided the original work is properly cited. 
as restlessness. Although concurrent use of a reliable pain scale and the PAED scale may decrease the error associated with pain, it does not guarantee clear differentiation between primary agitation and agitation due to pain.

Although the first cases of emergence agitation/delirium were reported almost half a century ago, the underlying etiology is still not fully understood. Further trials are needed to find clear strategies that prevent emergence agitation/delirium in children. We also need sensitive and specific tools to accurately differentiate the contributing factors of emergence agitation/ delirium.

\section{References}

1. Eckenhoff JE, Kneale DH, Dripps RD. The incidence and etiology of postanesthetic excitement. Anesthesiology 1961; 22: 667-73.

2. Voepel-Lewis T, Burke C. Differentiating pain and delirium is only part of assessing the agitated child. J Perianesth Nurs 2004; 19: 298-9.
3. Sikich N, Lerman J. Development and psychometric evaluation of the pediatric anesthesia emergence delirium scale. Anesthesiology 2004; 100: 1138-45.

4. Vlajkovic GP, Sindjelic RP. Emergence delirium in children: many questions, few answers. Anesth Analg 2007; 104: 84-91.

5. Welborn LG, Hannallah RS, Norden JM, Ruttimann UE, Callan CM. Comparison halothane in pediatric ambulatory patients. Anesth Analg 1996; 83: 917-20.

6. Kulka PJ, Bressem M, Tryba M. Clonidine prevents sevofluraneinduced agitation in children. Anesth Analg 2001; 93: 335-8.

7. Lerman J. Inhalation agents in pediatric anaesthesia - an update. Curr Opin Anaesthesiol 2007; 20: 221-6.

8. Lee CJ, Lee SE, Oh MK, Shin CM, Kim YJ, Choe YK, et al. The effect of propofol on the emergence agitation in children receiving sevoflurane for adenotonsillectomy. Korean J Anesthesiol 2010; 59: 75-81.

9. Dahmani S, Stany I, Brasher C, Lejeune C, Bruneau B, Wood C, et al. Pharmacological prevention of sevoflurane- and desflurane-related emergence agitation in children: a meta-analysis of published studies. Br J Anaesth 2010; 104: 216-23. 